S3 Raw data 


\section{Raw data for figure 1}


Figure $1 \mathrm{~A}$

Optical density

$\begin{array}{rrrrr}\text { gG1 (ng/ml } 1 \text { H435R T,G1 WT TA 3 R435H T,G3 WT TA99 } \\ 500 & 1.30255 & 1.2636 & 0.1026 & 0.15375 \\ 250 & 1.13465 & 1.13055 & 0.09375 & 0.1111 \\ 125 & 0.99555 & 0.91915 & 0.08905 & 0.0949 \\ 62.5 & 0.78325 & 0.68895 & 0.0942 & 0.09355 \\ 31.25 & 0.63555 & 0.4596 & 0.08865 & 0.0929 \\ 15.625 & 0.43975 & 0.299 & 0.0948 & 0.09235\end{array}$

Figure 1B

$\begin{array}{ccrrr}\text { Optical density } \\ \text { IgG3 } & \text { 1 H435R T,G1 WT TA@3 } & \text { R435H T,G3 WT TA99 } \\ 500 & 0.05165 & 0.0525 & 1.566 & 1.56775 \\ 250 & 0.04575 & 0.04515 & 1.519 & 1.52305 \\ 125 & 0.04505 & 0.0423 & 1.3019 & 1.3527 \\ 62.5 & 0.0442 & 0.04245 & 0.89315 & 1.0075 \\ 31.25 & 0.0435 & 0.04465 & 0.52185 & 0.65785 \\ 15.625 & 0.0448 & 0.04455 & 0.26055 & 0.3648\end{array}$

Figur 1C-D

Geometric mean on live cells (B16GP75) n=3 --> first sample is depicted in histogram concentration ug/ml

\begin{tabular}{|c|c|c|c|c|c|}
\hline \multicolumn{3}{|c|}{$\lg \mathrm{g} 1 \mathrm{wt}$} & \multicolumn{3}{|c|}{ IgG1 mut } \\
\hline 140 & 142 & 145 & 152 & 153 & 157 \\
\hline \multicolumn{3}{|c|}{$\operatorname{lgG} 3$ wt } & \multicolumn{3}{|c|}{ IgG3 mut } \\
\hline 154 & 154 & 158 & 158 & 159 & 163 \\
\hline \multicolumn{3}{|c|}{$\mathrm{mlgG} 2 \mathrm{a}$} & \multicolumn{3}{|c|}{ MG4 } \\
\hline 644 & 681 & 689 & 32.4 & 15 & 15.8 \\
\hline \multicolumn{3}{|c|}{ HEPC } & & & \\
\hline 6.74 & 6.82 & 6.57 & & & \\
\hline
\end{tabular}


Figure 1E

\begin{tabular}{|c|c|c|c|c|c|c|c|c|c|}
\hline \multirow{2}{*}{$\begin{array}{c}\text { concentré } \\
0.001\end{array}$} & \multicolumn{2}{|c|}{$\lg \mathrm{G} 1 \mathrm{wt}$} & \multicolumn{4}{|c|}{ IgG1 mut } & \multicolumn{3}{|c|}{$\operatorname{lgG} 3 \mathrm{wt}$} \\
\hline & 4 & 3.92 & 3.85 & 3.98 & 4.03 & 3.98 & 4.2 & 4.08 & 4.21 \\
\hline 0.01 & 7.16 & 7.33 & 7.28 & 10.1 & 9.97 & 9.38 & 8.17 & 7.9 & 7.9 \\
\hline 0.1 & 23 & 23.4 & 23.7 & 30.1 & 28.3 & 29.2 & 24.3 & 25 & 24.5 \\
\hline 1 & 98.4 & 95.3 & 92.2 & 115 & 112 & 110 & 105 & 102 & 99.3 \\
\hline 10 & 140 & 142 & 145 & 152 & 153 & 157 & 154 & 154 & 158 \\
\hline 100 & 148 & 148 & 146 & 160 & 162 & 164 & 166 & 168 & 167 \\
\hline \multicolumn{3}{|c|}{ lgG3 mut } & \multicolumn{3}{|c|}{ HEPC } & & & & \\
\hline 4.09 & 4.52 & 5.42 & 6.14 & 6.11 & 6.03 & & & & \\
\hline 9.53 & 9.75 & 9.66 & 4.75 & 4.87 & 4.76 & & & & \\
\hline 30.7 & 30.9 & 31.2 & 4.75 & 4.81 & 4.57 & & & & \\
\hline 117 & 116 & 114 & 4.69 & 4.68 & 4.66 & & & & \\
\hline 158 & 159 & 163 & 6.74 & 6.82 & 6.57 & & & & \\
\hline 168 & 172 & 174 & 8.19 & 7.91 & 11.2 & & & & \\
\hline
\end{tabular}

Figure $1 \mathrm{~F}$

concentr'

$\begin{array}{rrrrrrr}0.001 & 5.72 & 6.09 & 6.12 & 5.64 & 5.68 & 5.44 \\ 0.01 & 25.6 & 25 & 25.2 & 4.48 & 4.83 & 4.89 \\ 0.1 & 100 & 105 & 109 & 4.72 & 4.68 & 4.55 \\ 1 & 420 & 405 & 408 & 8.88 & 4.5 & 4.49 \\ 10 & 644 & 681 & 689 & 32.4 & 15 & 15.8 \\ 100 & 696 & 712 & 722 & 8.08 & 5.07 & 6.18\end{array}$

Geometric mean (live cells) $n=3$ 


\section{Raw data for figure 2}


$20111021 \mathrm{MB}$ fago B16.001 $1 \mu \mathrm{g} / \mathrm{ml}$ $20111021 \mathrm{MB}$ fago B16.002 $1 \mu \mathrm{g} / \mathrm{ml}$ $20111021 \mathrm{MB}$ fago B16.003 $1 \mu \mathrm{g} / \mathrm{ml}$ $20111021 \mathrm{MB}$ fago B16.004 $1 \mu \mathrm{g} / \mathrm{m}$ $20111021 \mathrm{MB}$ fago B16.005 $1 \mu \mathrm{g} / \mathrm{ml}$ $20111021 \mathrm{MB}$ fago B16.006 $1 \mu \mathrm{g} / \mathrm{m}$ $20111021 \mathrm{MB}$ fago B16.007 $1 \mu \mathrm{g} / \mathrm{ml}$ $20111021 \mathrm{MB}$ fago B16.008 $1 \mu \mathrm{g} / \mathrm{ml}$ $20111021 \mathrm{MB}$ fago B16.009 $1 \mu \mathrm{g} / \mathrm{ml}$ $20111021 \mathrm{MB}$ fago $B 16.0101 \mu \mathrm{g} / \mathrm{ml}$ $20111021 \mathrm{MB}$ fago B16.011 $1 \mu \mathrm{g} / \mathrm{m}$ $20111021 \mathrm{MB}$ fago B16.012 $1 \mu \mathrm{g} / \mathrm{m}$ $20111021 \mathrm{MB}$ fago B16.013 $1 \mu \mathrm{g} / \mathrm{ml}$ $20111021 \mathrm{MB}$ fago B16.014 $1 \mu \mathrm{g} / \mathrm{ml}$ $20111021 \mathrm{MB}$ fago B16.015 $1 \mu \mathrm{g} / \mathrm{ml}$ $20111021 \mathrm{MB}$ fago B16.016 $1 \mu \mathrm{g} / \mathrm{ml}$ $20111021 \mathrm{MB}$ fago B16.017 $1 \mu \mathrm{g} / \mathrm{ml}$ $20111021 \mathrm{MB}$ fago $\mathrm{B} 16.0181 \mu \mathrm{g} / \mathrm{ml}$ $20111021 \mathrm{MB}$ fago B16.019 $1 \mu \mathrm{g} / \mathrm{ml}$ $20111021 \mathrm{MB}$ fago B16.020 $1 \mu \mathrm{g} / \mathrm{ml}$ $20111021 \mathrm{MB}$ fago $B 16.0211 \mu \mathrm{g} / \mathrm{ml}$ $20111021 \mathrm{MB}$ fago B16.022 $1 \mu \mathrm{g} / \mathrm{ml}$ $20111021 \mathrm{MB}$ fago $\mathrm{B} 16.0231 \mu \mathrm{g} / \mathrm{ml}$ $20111021 \mathrm{MB}$ fago B16.026 0,1 $\mathrm{\mu g} / \mathrm{ml}$ IgG1 wt $20111021 \mathrm{MB}$ fago $\mathrm{B} 16.027 \mathrm{0,1} \mu \mathrm{g} / \mathrm{ml} \quad \mathrm{lgG} 1 \mathrm{H} 435 \mathrm{R}$ $20111021 \mathrm{MB}$ fago B16.028 0,1 $\mathrm{gg} / \mathrm{ml}$ IgG1 H435R $20111021 \mathrm{MB}$ fago B16.029 0,1 $\mathrm{gg} / \mathrm{ml}$ IgG1 H435R

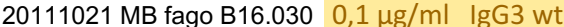
$20111021 \mathrm{MB}$ fago B16.031 0,1 $\mathrm{gg} / \mathrm{ml}$ lgG3 wt $20111021 \mathrm{MB}$ fago B16.032 0,1 $\mathrm{gg} / \mathrm{ml}$ lgG3 wt $20111021 \mathrm{MB}$ fago B16.033 0,1 $\mathrm{gg} / \mathrm{ml}$ IgG3 R435H $20111021 \mathrm{MB}$ fago B16.034 0,1 $\mathrm{gg} / \mathrm{ml}$ IgG3 R435H $20111021 \mathrm{MB}$ fago B16.035 0,1 $\mathrm{kg} / \mathrm{ml}$ IgG3 R435H $20111021 \mathrm{MB}$ fago $\mathrm{B} 16.0360,1 \mu \mathrm{g} / \mathrm{ml} \mathrm{mlgG} 2 \mathrm{a}$ $20111021 \mathrm{MB}$ fago $B 16.0370,1 \mu \mathrm{g} / \mathrm{ml} \mathrm{mlgG} 2 \mathrm{a}$ $20111021 \mathrm{MB}$ fago $\mathrm{B} 16.0380,1 \mu \mathrm{g} / \mathrm{ml} \mathrm{mlgG} 2 \mathrm{a}$ $20111021 \mathrm{MB}$ fago B16.039 $0,1 \mu \mathrm{g} / \mathrm{ml} \mathrm{MG} 4$ $20111021 \mathrm{MB}$ fago B16.040 0,1 $\mathrm{\mu g} / \mathrm{ml}$ MG4 $20111021 \mathrm{MB}$ fago B16.041 $0,1 \mu \mathrm{g} / \mathrm{ml}$ MG4 $20111021 \mathrm{MB}$ fago B16.042 0,1 $\mathrm{\mu g} / \mathrm{ml}$ HEPC $20111021 \mathrm{MB}$ fago B16.043 0,1 $\mathrm{mg} / \mathrm{ml}$ HEPC $20111021 \mathrm{MB}$ fago B16.044 0,1 $\mu \mathrm{g} / \mathrm{ml} \mathrm{HEPC}$ $20111021 \mathrm{MB}$ fago B16.045 0,1 $\mathrm{gg} / \mathrm{ml}$ no a.b. $20111021 \mathrm{MB}$ fago B16.046 0,1 $\mathrm{gg} / \mathrm{ml}$ no a.b. $20111021 \mathrm{MB}$ fago B16.047 0,1 $\mathrm{gg} / \mathrm{ml}$ no a.b. $20111021 \mathrm{MB}$ fago B16.048 $0,01 \mu \mathrm{g} / \mathrm{ml} \quad \mathrm{lgG} 1 \mathrm{wt}$ $20111021 \mathrm{MB}$ fago B16.049 $0,01 \mu \mathrm{g} / \mathrm{ml} \quad \mathrm{lgG} 1 \mathrm{wt}$ $20111021 \mathrm{MB}$ fago B16.050 $0,01 \mu \mathrm{g} / \mathrm{ml} \quad \mathrm{lgG} 1 \mathrm{wt}$ $20111021 \mathrm{MB}$ fago B16.051 0,01 $\mathrm{gg} / \mathrm{ml} \quad \mathrm{lgG} 1 \mathrm{H} 435 \mathrm{R}$ $20111021 \mathrm{MB}$ fago B16.052 $0,01 \mu \mathrm{g} / \mathrm{ml}$ lgG1 H435R $20111021 \mathrm{MB}$ fago B16.053 $0,01 \mu \mathrm{g} / \mathrm{ml} \quad \mathrm{lgG} 1 \mathrm{H} 435 \mathrm{R}$ $20111021 \mathrm{MB}$ fago B16.054 $0,01 \mu \mathrm{g} / \mathrm{ml} \quad \mathrm{lgG} 3 \mathrm{wt}$ $20111021 \mathrm{MB}$ fago B16.055 $0,01 \mu \mathrm{g} / \mathrm{ml} \quad \mathrm{lgG} 3 \mathrm{wt}$ $20111021 \mathrm{MB}$ fago B16.056 $0,01 \mu \mathrm{g} / \mathrm{ml} \quad \mathrm{lgG} 3 \mathrm{wt}$ $20111021 \mathrm{MB}$ fago B16.057 $0,01 \mu \mathrm{g} / \mathrm{ml} \quad \lg \mathrm{g} 3 \mathrm{R} 435 \mathrm{H}$ $20111021 \mathrm{MB}$ fago B16.058 $0,01 \mu \mathrm{g} / \mathrm{ml} \quad \mathrm{lgG} 3 \mathrm{R} 435 \mathrm{H}$ $20111021 \mathrm{MB}$ fago B16.059 $0,01 \mu \mathrm{g} / \mathrm{ml} \quad \mathrm{lgG} 3 \mathrm{R} 435 \mathrm{H}$ $20111021 \mathrm{MB}$ fago B16.060 $0,01 \mu \mathrm{g} / \mathrm{ml} \quad \mathrm{mlgG} 2 \mathrm{a}$ $20111021 \mathrm{MB}$ fago B16.061 $0,01 \mu \mathrm{g} / \mathrm{ml} \mathrm{mlgG} 2 \mathrm{a}$ $20111021 \mathrm{MB}$ fago B16.062 $0,01 \mu \mathrm{g} / \mathrm{ml} \mathrm{mlgG} 2 \mathrm{a}$ $20111021 \mathrm{MB}$ fago B16.063 $0,01 \mu \mathrm{g} / \mathrm{ml} \quad$ MG4 $20111021 \mathrm{MB}$ fago B16.064 $0,01 \mu \mathrm{g} / \mathrm{ml}$ MG4 $20111021 \mathrm{MB}$ fago B16.065 $0,01 \mu \mathrm{g} / \mathrm{ml}$ MG4 $20111021 \mathrm{MB}$ fago B16.066 $0,01 \mu \mathrm{g} / \mathrm{ml}$ HEPC $20111021 \mathrm{MB}$ fago B16.067 $0,01 \mu \mathrm{g} / \mathrm{ml}$ HEPC $20111021 \mathrm{MB}$ fago B16.068 $0,01 \mu \mathrm{g} / \mathrm{ml}$ HEPC $20111021 \mathrm{MB}$ fago $B 16.0690,01 \mu \mathrm{g} / \mathrm{ml}$ no a.b. $20111021 \mathrm{MB}$ fago B16.070 $0,01 \mu \mathrm{g} / \mathrm{ml}$ no a.b. $20111021 \mathrm{MB}$ fago B16.071 $0,01 \mu \mathrm{g} / \mathrm{ml}$ no a.b. $20111021 \mathrm{MB}$ fago B16.072 $20111021 \mathrm{MB}$ fago B16.073 $20111021 \mathrm{MB}$ fago B16.074 $20111021 \mathrm{MB}$ fago B16.075 Mean

SD excl debris | Freq. of Parent excl debris/B10F10 | Freq. of Parent excl debris/double | Freq. of Parent $\begin{array}{rrr}82,4 & 8,84 & 6,11 \\ 84,5 & 9,63 & 4,4 \\ 85,7 & 11,2 & 6,53 \\ 84,5 & 9,37 & 6,6 \\ 85 & 9,28 & 6,31 \\ 85,2 & 10,5 & 6,5 \\ 87,1 & 17,8 & 5,05 \\ 86,4 & 18,5 & 4,87 \\ 86,2 & 18,4 & 5,6 \\ 86,7 & 15,2 & 4,71 \\ 87,9 & 19,9 & \end{array}$

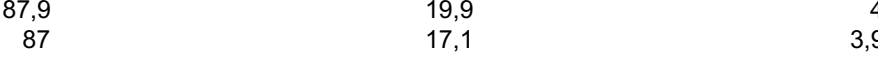

$86,8 \quad 7,35 \quad 7,92$

$87,1 \quad 9,22 \quad 6,61$

$\begin{array}{lll}87,3 & 8,33 & 7,09\end{array}$

$\begin{array}{lll}87 & 17,6 & 3,42\end{array}$

$\begin{array}{rrr}88,6 & 22,4 & 4 \\ 87,9 & 20,5 & 3,5\end{array}$

$\begin{array}{lll}86,8 & 20,8 & 3,33\end{array}$

$\begin{array}{lll}87,6 & 20,9 & 3,44\end{array}$

$88,2 \quad 21,3 \quad 3,26$

$89 \quad 21,7 \quad 3,08$

$\begin{array}{rrr}85,4 & 17,4 & 2,96 \\ 87,2 & 16 & 3,97\end{array}$

$\begin{array}{lll}87,3 & 18,1 & 4,14 \\ 88,5 & 18,2 & 4,98\end{array}$

$87,7 \quad 15,3 \quad 4,17$

$88,9 \quad 16,4 \quad 4,34$

$86,7 \quad 16,4 \quad 4,14$

$88,6 \quad 18,8 \quad 3,41$

$\begin{array}{lll}89,6 & 17,2 & 2,65\end{array}$

$88,1 \quad 20,2 \quad 4,55$

$88 \quad 15,8 \quad 3,63$

$\begin{array}{lll}87,6 & 17,4 & 3,33\end{array}$

$88,4 \quad 17,2 \quad 3,12$

$87,5 \quad 15,3 \quad 3,4$

$87,9 \quad 18,6 \quad 3,08$

$89,1 \quad 18,6 \quad 4,39$

$89,1 \quad 19,1 \quad 3,15$

$89,3 \quad 20,1 \quad 1,62$

$\begin{array}{lll}88,9 & 19,8 & 2,74\end{array}$

2,86

$88,3 \quad 20,6 \quad 2,09$

$88,4 \quad 20 \quad 1,91$

$\begin{array}{llr}87 & 19,2 & 2,27\end{array}$

$86,9 \quad 18,3 \quad 1,8$

$88,2 \quad 20 \quad 2,43$

$\begin{array}{llr}87,3 & 22,6 & 1,79\end{array}$

$\begin{array}{rr}87,7 & 22,6 \\ 86,5 & 1,7\end{array}$

$86,5 \quad 21,2 \quad 1,91$

$86,7 \quad 16,6 \quad 1,56$

$87,6 \quad 22,6 \quad 2,94$

$88,8 \quad 20,8 \quad 1,9$

$87,4 \quad 20,4 \quad 1,64$

21

$87,2 \quad 24,5 \quad 2,42$

$85,1 \quad 17,5 \quad 2,39$

$85,5 \quad 18,8 \quad 2,31$

$\begin{array}{lll}84,5 & 15,5 & 1,59\end{array}$

86,1

$86,3 \quad 16,8$

87,2

85,4

85,9

87,6

85,9

85,8

84,8

85,4

86,2

85,8

88,2

67,8

96,6

96,7

87,1

3,06

3
57 7

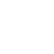

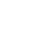

.




$\begin{array}{lr}\text { double } & \text { average } \\ \text { lgG1 wt } & 5,68 \\ \text { lgG1 mut } & 6,513333 \\ \text { lgG3 wt } & 5,18 \\ \text { lgG3 mut } & 4,203333 \\ \text { mlgG2a } & 7,206667 \\ \text { HEPC } & 3,343333 \\ \text { MG4 } & 3,64 \\ \text { no mAb } & 3,02\end{array}$

$\begin{array}{lr}\text { double } & \text { average } \\ \text { IgG1 wt } & 4,363333 \\ \text { IgG1 mut } & 4,216667 \\ \text { IgG3 wt } & 3,536667 \\ \text { IgG3 mut } & 3,36 \\ \text { mlgG2a } & 3,623333 \\ \text { HEPC } & 2,286667 \\ \text { MG4 } & 2,503333 \\ \text { no mAb } & 2,166667\end{array}$

\begin{tabular}{|c|c|}
\hline B16 & \multirow[t]{9}{*}{$1 u$} \\
\hline lgG1 wt & \\
\hline lgG1 mut & \\
\hline $\operatorname{lgG} 3$ wt & \\
\hline lgG3 mut & \\
\hline mlgG2a & \\
\hline HEPC & \\
\hline MG4 & \\
\hline no mAb & \\
\hline double & \\
\hline $\operatorname{lgG} 1$ wt & \\
\hline lgG1 mut & \\
\hline $\operatorname{lgG} 3$ wt & \\
\hline lgG3 mut & \\
\hline mlgG2a & \\
\hline HEPC & \\
\hline MG4 & \\
\hline no mAb & \\
\hline
\end{tabular}

$\begin{array}{ll}8,84 & 9,63 \\ 9,37 & 9,28 \\ 17,8 & 18,5 \\ 15,2 & 19,9 \\ 7,35 & 9,22 \\ 20,8 & 20,9 \\ 17,6 & 22,4 \\ 21,7 & 17,4\end{array}$

\begin{tabular}{lrrr} 
& & & \multicolumn{2}{c}{ HepC=100\% } \\
11,2 & 42,09524 & 45,85714 & 53,33333 \\
10,5 & 44,61905 & 44,19048 & 50 \\
18,4 & 84,7619 & 88,09524 & 87,61905 \\
17,1 & 72,38095 & 94,7619 & 81,42857 \\
8,33 & 35 & 43,90476 & 39,66667 \\
21,3 & 99,04762 & 99,52381 & 101,4286 \\
20,5 & 83,80952 & 106,6667 & 97,61905 \\
& 103,3333 & 82,85714 & 0
\end{tabular}

\begin{tabular}{lrrr} 
B16 & 0,1 ug & & \\
\hline IgG1 wt & 16 & 18,1 & 18,2 \\
IgG1 mut & 15,3 & 16,4 & 16,4 \\
IgG3 wt & 18,8 & 17,2 & 20,2 \\
IgG3 mut & 15,8 & 17,4 & 17,2 \\
mlgG2a & 15,3 & 18,6 & 18,6 \\
HEPC & 21,3 & 20,6 & 20 \\
MG4 & 19,1 & 20,1 & 19,8 \\
no mAb & 19,2 & 18,3 & 20 \\
\hline & & & \\
double & & & \\
IgG1 wt & 3,97 & 4,14 & 4,98 \\
IgG1 mut & 4,17 & 4,34 & 4,14 \\
IgG3 wt & 3,41 & 2,65 & 4,55 \\
IgG3 mut & 3,63 & 3,33 & 3,12 \\
mlgG2a & 3,4 & 3,08 & 4,39 \\
HEPC & 2,86 & 2,09 & 1,91 \\
MG4 & 3,15 & 1,62 & 2,74 \\
no mAb & 2,27 & 1,8 & 2,43
\end{tabular}

HepC=1

6,53

6,57

5,62

7,09

3,26

3,5

$\begin{array}{rrr} & & \\ \text { HepC=1 } \\ 1,827517 & 1,316052 & 1,953141 \\ 1,992024 & 1,887338 & 1,965105 \\ 1,510469 & 1,45663 & 1,680957 \\ 1,408774 & 1,196411 & 1,1665 \\ 2,368893 & 1,977069 & 2,120638 \\ 0,996012 & 1,028913 & 0,975075 \\ 1,022931 & 1,196411 & 1,046859 \\ 0,921236 & 0,885344 & 0\end{array}$

3,343333

20,63333

2,286667

\begin{tabular}{lrrrrrr} 
B16 & 0,01 & & & & & HepC=100\% \\
IgG1 wt & 22,6 & 22,6 & 21,2 & 114,7208 & 114,7208 & 107,6142 \\
IgG1 mut & 16,6 & 22,6 & 20,8 & 84,26396 & 114,7208 & 105,5838 \\
IgG3 wt & 20,4 & 21 & 24,5 & 103,5533 & 106,599 & 124,3655 \\
IgG3 mut & 17,5 & 18,8 & 15,5 & 88,83249 & 95,43147 & 78,6802 \\
mlgG2a & 18,4 & 16,8 & 21,3 & 93,40102 & 85,27919 & 108,1218 \\
HEPC & 21,2 & 19 & 18,9 & 107,6142 & 96,4467 & 95,93909 \\
MG4 & 17,4 & 19,6 & 21,7 & 88,32487 & 99,49239 & 110,1523 \\
no mAb & 15,9 & 21,1 & 17,5 & 80,71066 & 107,1066 & 88,83249 \\
\hline & & & & & & \\
double & & & & & & HepC=1 \\
IgG1 wt & 1,79 & 1,7 & 1,91 & 0,727642 & 0,691057 & 0,776423 \\
IgG1 mut & 1,56 & 2,94 & 1,9 & 0,634146 & 1,195122 & 0,772358 \\
lgG3 wt & 1,64 & 2,57 & 2,42 & 0,666667 & 1,044715 & 0,98374 \\
IgG3 mut & 2,39 & 2,31 & 1,59 & 0,971545 & 0,939024 & 0,646341 \\
mlgG2a & 1,98 & 1,9 & 2 & 0,804878 & 0,772358 & 0,813008 \\
HEPC & 2,62 & 4,18 & 2,3 & 1,065041 & 1,699187 & 0,934959 \\
MG4 & 2,44 & 17,2 & 3,11 & 0,99187 & 6,99187 & 1,264228 \\
no mAb & 2,03 & 3,88 & 3,94 & 0,825203 & 1,577236 & 1,601626 \\
\hline
\end{tabular}




\section{Raw data for figure 3}


MCB12-26 exp 8

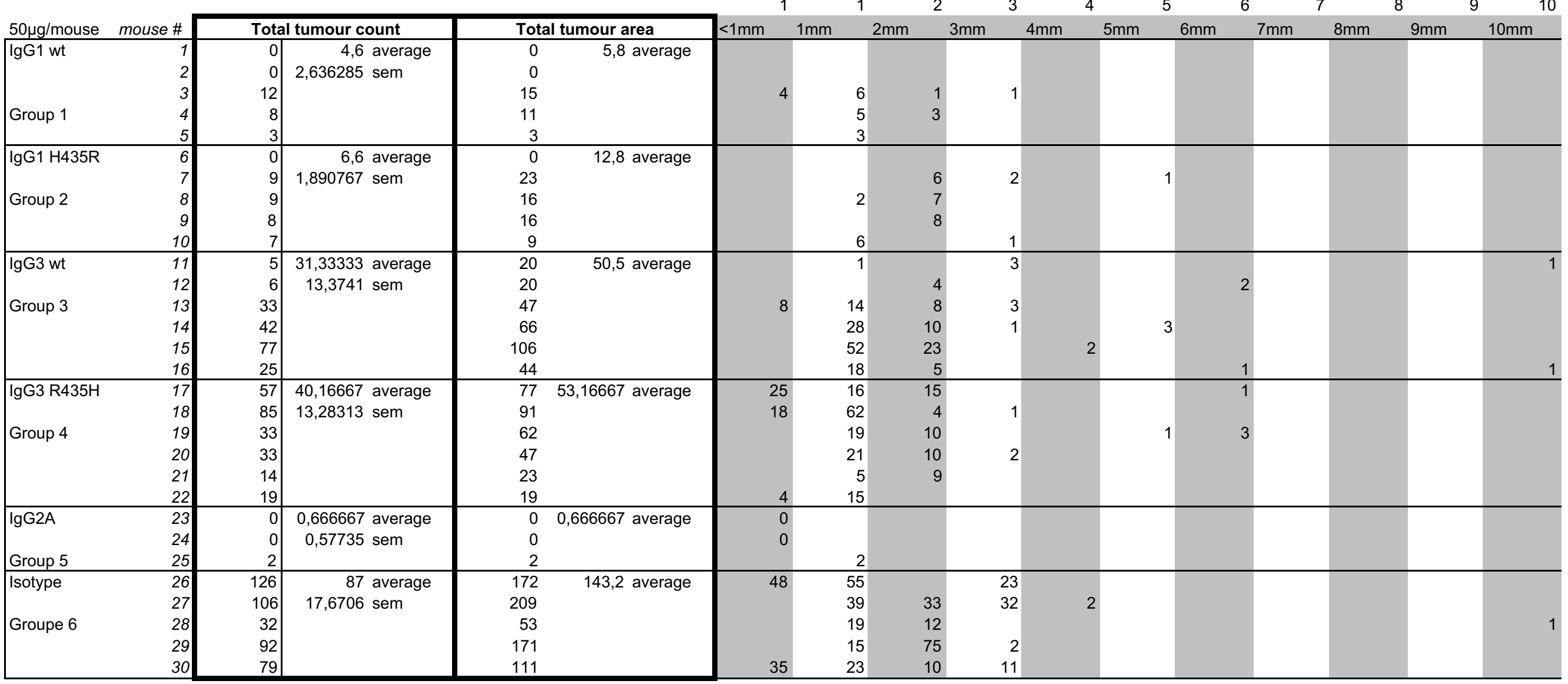




\section{Raw data for sup figure $2 \mathrm{~A}$}




\begin{tabular}{|c|c|c|c|c|c|c|c|c|c|c|c|}
\hline & \multirow[b]{2}{*}{$\mathrm{mAb}$ conc (ug/ml) } & \multicolumn{5}{|c|}{ With PMN } & \multicolumn{5}{|c|}{ Without PMN } \\
\hline & & no $\mathrm{mAb}$ & 2 & 1 & 0,1 & 0,01 & no $\mathrm{mAb}$ & 2 & 1 & 0,1 & 0,01 \\
\hline & no $\mathrm{mAb}$ & $\begin{array}{r}42797,33 \\
39788,33 \\
39266,33\end{array}$ & & & & & $\begin{array}{l}34893,33 \\
33928,33 \\
34388,33\end{array}$ & & & & \\
\hline & IgG1 TNP & & 49222,33 & 38162,33 & 40414,33 & 38953,33 & & 35852,33 & 31951,33 & 32980,33 & 35607,33 \\
\hline & & & 42329,33 & 41810,33 & 36105,33 & 39250,33 & & 35238,33 & 35180,33 & 32843,33 & 34797,33 \\
\hline & & & 42811,33 & 36849,33 & 33626,33 & 36599,33 & & 38301,33 & 35649,33 & 33113,33 & 33587,33 \\
\hline & $\lg G 3$ TNP & & 37654,33 & 36424,33 & 36791,33 & 34803,33 & & 36629,33 & 34231,33 & 33813,33 & 35149,33 \\
\hline & & & 40276,33 & 38982,33 & 39327,33 & 37428,33 & & 36993,33 & 35710,33 & 35787,33 & 35080,33 \\
\hline & & & 42476,33 & 36603,33 & 37961,33 & 37434,33 & & 38921,33 & 37486,33 & 39311,33 & 38846,33 \\
\hline & Serum IgA & & 33561,33 & 34457,33 & 36453,33 & 35372,33 & & 34158,33 & 33724,33 & 32989,33 & 34005,33 \\
\hline & & & 35437,33 & 36797,33 & 34285,33 & 41166,33 & & 33347,33 & 33490,33 & 33253,33 & 32960,33 \\
\hline & & & 36563,33 & 39457,33 & 41145,33 & 36802,33 & & 36919,33 & 38084,33 & 37381,33 & 37382,33 \\
\hline & $\lg$ A TA99 & & 36018,33 & 38943,33 & 44727,33 & 44723,33 & & 36168,33 & 34922,33 & 33819,33 & 33985,33 \\
\hline & & & 33544,33 & 35826,33 & 39870,33 & 36245,33 & & 33336,33 & 33982,33 & 33722,33 & 32903,33 \\
\hline Plate 2/4 & & & 29972,33 & 32648,33 & 36225,33 & 35935,33 & & 33283,33 & 32458,33 & 34343,33 & 35972,33 \\
\hline & no $m A b$ & 38071,33 & & & & & 32374,67 & & & & \\
\hline & & 31617,33 & & & & & 32024,67 & & & & \\
\hline & & 32758,33 & & & & & 31400,67 & & & & \\
\hline & $\lg G 1 \mathrm{wt}$ & & 28746,33 & 27745,33 & 29178,33 & 29441,33 & & 35772,67 & 33030,67 & 33516,67 & 32168,67 \\
\hline & & & 35815,33 & 33617,33 & 32623,33 & 32055,33 & & 34187,67 & 31710,67 & 31906,67 & 32446,67 \\
\hline & & & 39773,33 & 34636,33 & 34896,33 & 33457,33 & & 34782,67 & 30788,67 & 30112,67 & 29150,67 \\
\hline & $\operatorname{lgG} 1 \mathrm{H} 435 \mathrm{R}$ & & 36484,33 & 35006,33 & 35512,33 & 37226,33 & & 33367,67 & 30479,67 & 29847,67 & 30154,67 \\
\hline & & & 37396,33 & 38309,33 & 33864,33 & 33759,33 & & 33800,67 & 31029,67 & 30832,67 & 31154,67 \\
\hline & & & 36584,33 & 36353,33 & 35882,33 & 35587,33 & & 34120,67 & 34339,67 & 33827,67 & 35530,67 \\
\hline & $\operatorname{lgG} 3$ wt & & 35305,33 & 34577,33 & 34569,33 & 32410,33 & & 28870,67 & 30445,67 & 29903,67 & 30818,67 \\
\hline & & & 34434,33 & 33252,33 & 32198,33 & 33412,33 & & 31147,67 & 30026,67 & 30617,67 & 30020,67 \\
\hline & & & 34688,33 & 37914,33 & 34533,33 & 34603,33 & & 33296,67 & 33166,67 & 33834,67 & 33711,67 \\
\hline & $\operatorname{lgG} 3 \mathrm{R} 435 \mathrm{H}$ & & 30731,33 & 35479,33 & 38266,33 & 36210,33 & & 30896,67 & 32198,67 & 32072,67 & 31802,67 \\
\hline & & & 33688,33 & 30473,33 & 32179,33 & 31783,33 & & 30905,67 & 29912,67 & 29264,67 & 30967,67 \\
\hline Plate $1 / 3$ & & & 32603,33 & 32134,33 & 30147,33 & 31392,33 & & 28495,67 & 28961,67 & 30118,67 & 30461,67 \\
\hline & no mAb Average & plate 1 & 34149,00 & & & & & & & & \\
\hline & & plate 2 & 40617,33 & & & & & & & & \\
\hline & & plate 3 & 31933,33 & & & & & & & & \\
\hline & & plate 4 & 34403,33 & & & & & & & & \\
\hline
\end{tabular}

Relative to no mAb

\begin{tabular}{|c|c|c|c|c|c|c|c|c|c|c|c|}
\hline & \multirow[b]{2}{*}{$\mathrm{mAb}$ conc (ug/ml) } & \multicolumn{5}{|c|}{ With PMN } & \multicolumn{5}{|c|}{ Without PMN } \\
\hline & & no $\mathrm{mAb}$ & 2 & 1 & 0,1 & 0,01 & no $\mathrm{mAb}$ & 2 & 1 & 0,1 & 0,01 \\
\hline & no $\mathrm{mAb}$ & $\begin{array}{r}105,37 \\
97,96 \\
96,67\end{array}$ & & & & & $\begin{array}{r}101,42 \\
98,62 \\
99,96\end{array}$ & & & & \\
\hline & $\lg 1$ TNP & & 121,19 & 93,96 & 99,50 & 95,90 & & 104,21 & 92,87 & 95,86 & 103,50 \\
\hline & & & 104,21 & 102,94 & 88,89 & 96,63 & & 102,43 & 102,26 & 95,47 & 101,15 \\
\hline & & & 105,40 & 90,72 & 82,79 & 90,11 & & 111,33 & 103,62 & 96,25 & 97,63 \\
\hline & $\operatorname{lgG} 3$ TNP & & 92,71 & 89,68 & 90,58 & 85,69 & & 106,47 & 99,50 & 98,29 & 102,17 \\
\hline & & & 99,16 & 95,97 & 96,82 & 92,15 & & 107,53 & 103,80 & 104,02 & 101,97 \\
\hline & & & 104,58 & 90,12 & 93,46 & 92,16 & & 113,13 & 108,96 & 114,27 & 112,91 \\
\hline & Serum IgA & & 82,63 & 84,83 & 89,75 & 87,09 & & 99,29 & 98,03 & 95,89 & 98,84 \\
\hline & & & 87,25 & 90,60 & 84,41 & 101,35 & & 96,93 & 97,35 & 96,66 & 95,81 \\
\hline & & & 90,02 & 97,14 & 101,30 & 90,61 & & 107,31 & 110,70 & 108,66 & 108,66 \\
\hline & $\lg A$ TA99 & & 88,68 & 95,88 & 110,12 & 110,11 & & 105,13 & 101,51 & 98,30 & 98,79 \\
\hline & & & 82,59 & 88,20 & 98,16 & 89,24 & & 96,90 & 98,78 & 98,02 & 95,64 \\
\hline Plate 2/4 & & & 73,79 & 80,38 & 89,19 & 88,47 & & 96,74 & 94,35 & 99,83 & 104,56 \\
\hline & no $m A b$ & 111,49 & & & & & 101,38 & & & & \\
\hline & & 92,59 & & & & & 100,29 & & & & \\
\hline & & 95,93 & & & & & 98,33 & & & & \\
\hline & $\operatorname{lgG} 1 \mathrm{wt}$ & & 84,18 & 81,25 & 85,44 & 86,21 & & 112,02 & 103,44 & 104,96 & 100,74 \\
\hline & & & 104,88 & 98,44 & 95,53 & 93,87 & & 107,06 & 99,30 & 99,92 & 101,61 \\
\hline & & & 116,47 & 101,43 & 102,19 & 97,97 & & 108,92 & 96,42 & 94,30 & 91,29 \\
\hline & IgG1 H435R & & 106,84 & 102,51 & 103,99 & 109,01 & & 104,49 & 95,45 & 93,47 & 94,43 \\
\hline & & & 109,51 & 112,18 & 99,17 & 98,86 & & 105,85 & 97,17 & 96,55 & 97,56 \\
\hline & & & 107,13 & 106,46 & 105,08 & $104,21 \|$ & & 106,85 & 107,54 & 105,93 & 111,27 \\
\hline & $\operatorname{lgG} 3 w t$ & & 103,39 & 101,25 & 101,23 & 94,91 & & 90,41 & 95,34 & 93,64 & 96,51 \\
\hline & & & 100,84 & 97,37 & 94,29 & 97,84 & & 97,54 & 94,03 & 95,88 & 94,01 \\
\hline & & & 101,58 & 111,03 & 101,13 & 101,33 & & 104,27 & 103,86 & 105,95 & 105,57 \\
\hline & IgG3 R435H & & 89,99 & 103,90 & 112,06 & $106,04 \|$ & & 96,75 & 100,83 & 100,44 & 99,59 \\
\hline & & & 98,65 & 89,24 & 94,23 & 93,07 & & 96,78 & 93,67 & 91,64 & 96,98 \\
\hline Plate $1 / 3$ & & & 95,47 & 94,10 & 88,28 & 91,93 & & 89,23 & 90,69 & 94,32 & 95,39 \\
\hline
\end{tabular}




\begin{tabular}{rrrrrrrrrr}
\hline With PMN & no mAb & Serum IgA & IgA TA99 & \multicolumn{1}{l}{ IgG1 TNP } & IgG3 TNP & IgG1 wt & \multicolumn{1}{l}{ IgG1 H435FlgG3 wt } & IgG3 R435H \\
105,37 & 82,63 & 88,68 & 121,19 & 92,71 & & & & \\
97,96 & 87,25 & 82,59 & 104,21 & 99,16 & & & & \\
96,67 & 90,02 & 73,79 & 105,40 & 104,58 & & & \\
111,49 & & & & & 84,18 & 106,84 & 103,39 & 89,99 \\
92,59 & & & & & 104,88 & 109,51 & 100,84 & 98,65 \\
95,93 & & & & & 116,47 & 107,13 & 101,58 & 95,47
\end{tabular}

1ug/ml

\begin{tabular}{|c|c|c|c|c|c|c|c|c|c|}
\hline \multirow[t]{7}{*}{ With PMN } & \multirow[t]{4}{*}{ no $\mathrm{mAb}$} & \multicolumn{2}{|c|}{ Serum IgA IgA TA99 } & IgG1 TNP & \multicolumn{2}{|c|}{ IgG3 TNP IgG1 wt } & \multicolumn{2}{|c|}{$\operatorname{lgG} 1 \mathrm{H} 435 \mathrm{~F} \operatorname{lgG} 3 \mathrm{wt}$} & \multirow[t]{2}{*}{$\lg \mathrm{G} 3 \mathrm{R} 435 \mathrm{H}$} \\
\hline & & 84,83 & 95,88 & 93,96 & 89,68 & & & & \\
\hline & & 90,60 & 88,20 & 102,94 & 95,97 & & & & \\
\hline & & 97,14 & 80,38 & 90,72 & 90,12 & & & & \\
\hline & & & & & & 81,25 & 102,51 & 101,25 & 103,90 \\
\hline & & & & & & 98,44 & 112,18 & 97,37 & 89,24 \\
\hline & & & & & & 101,43 & 106,46 & 111,03 & 94,10 \\
\hline
\end{tabular}

$0,1 \mathrm{ug} / \mathrm{ml}$

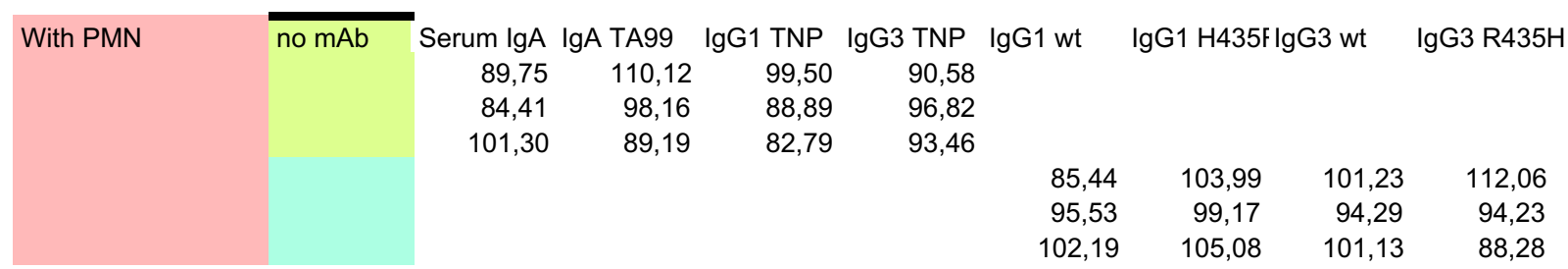

$0,01 \mathrm{ug} / \mathrm{ml}$

\begin{tabular}{|c|c|c|c|c|c|c|c|c|c|}
\hline \multirow[t]{7}{*}{ With PMN } & \multirow[t]{7}{*}{ no $m A b$} & \multirow{4}{*}{$\begin{array}{r}\text { Serum IgA } \\
87,09 \\
101,35 \\
90,61\end{array}$} & \multirow{3}{*}{$\begin{array}{r}\text { IgA TA99 } \\
110,11 \\
89,24\end{array}$} & \multirow{2}{*}{$\begin{array}{r}\operatorname{lgG} 1 \text { TNP } \\
95,90\end{array}$} & IgG3 TNP & \multirow{3}{*}{$\lg \mathrm{l} 1 \mathrm{wt}$} & \multicolumn{2}{|c|}{ IgG1 H435F IgG3 wt } & \multirow[t]{4}{*}{$\lg$ G3 R435H } \\
\hline & & & & & 85,69 & & & & \\
\hline & & & & 96,63 & 92,15 & & & & \\
\hline & & & 88,47 & 90,11 & 92,16 & & & & \\
\hline & & & & & & 86,21 & 109,01 & 94,91 & 106,04 \\
\hline & & & & & & 93,87 & 98,86 & 97,84 & 93,07 \\
\hline & & & & & & 97,97 & 104,21 & 101,33 & 91,93 \\
\hline
\end{tabular}

$2 u g / m l$

Without PMN

\begin{tabular}{rrrrrrrrr}
\hline no $\mathrm{mAb}$ & Serum IgA & IgA TA99 & IgG1 TNP & IgG3 TNP & IgG1 wt & IgG1 H435FlgG3 wt & IgG3 R435H \\
101,42 & 99,29 & 105,13 & 104,21 & 106,47 & & & & \\
98,62 & 96,93 & 96,90 & 102,43 & 107,53 & & & & \\
99,96 & 107,31 & 96,74 & 111,33 & 113,13 & & & & \\
101,38 & & & & & 112,02 & 104,49 & 90,41 & 96,75 \\
100,29 & & & & & 107,06 & 105,85 & 97,54 & 96,78 \\
98,33 & & & & & 108,92 & 106,85 & 104,27 & 89,23
\end{tabular}

$1 \mathrm{ug} / \mathrm{ml}$

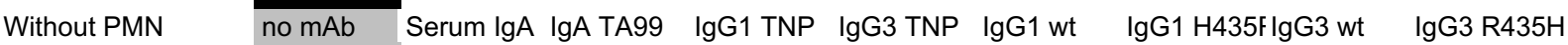
$\begin{array}{lll}98,03 & 101,51 \quad 92,87 & 99,50\end{array}$

$\begin{array}{rrrr}97,35 & 98,78 & 102,26 & 103,80 \\ 110,70 & 94,35 & 103,62 & 108,96\end{array}$

$\begin{array}{rrrr}103,44 & 95,45 & 95,34 & 100,83 \\ 99,30 & 97,17 & 94,03 & 93,67 \\ 96,42 & 107,54 & 103,86 & 90,69\end{array}$

$0,1 \mathrm{ug} / \mathrm{ml}$

\begin{tabular}{|c|c|c|c|c|c|c|c|c|c|}
\hline \multirow[t]{7}{*}{ Without PMN } & \multirow[t]{4}{*}{ no $\mathrm{mAb}$} & \multirow{4}{*}{$\begin{array}{r}\text { Serum IgA } \\
95,89 \\
96,66 \\
108,66\end{array}$} & TA99 & \multirow{2}{*}{$\begin{array}{r}\operatorname{lgG} 1 \text { TNP } \\
95,86\end{array}$} & \multirow{2}{*}{$\begin{array}{r}\lg 33 \text { TNP } \\
98,29\end{array}$} & \multirow[t]{3}{*}{$\operatorname{lgG} 1 \mathrm{wt}$} & \multicolumn{2}{|c|}{$\operatorname{lgG} 1 \mathrm{H} 435 \mathrm{FlgG} 3 \mathrm{wt}$} & \multirow[t]{2}{*}{ IgG3 R435H } \\
\hline & & & 98,30 & & & & & & \\
\hline & & & 98,02 & 95,47 & 104,02 & & & & \\
\hline & & & 99,83 & 96,25 & 114,27 & & & & \\
\hline & & & & & & 104,96 & 93,47 & 93,64 & 100,44 \\
\hline & & & & & & 99,92 & 96,55 & 95,88 & 91,64 \\
\hline & & & & & & 94,30 & 105,93 & 105,95 & 94,32 \\
\hline
\end{tabular}

$0,01 \mathrm{ug} / \mathrm{ml}$

Without PMN

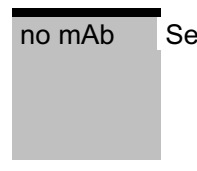

$\begin{array}{rrrr}\text { Serum IgA } & \text { IgA TA99 } & \text { IgG1 TNP } & \text { IgG3 TNP } \\ 98,84 & 98,79 & 103,50 & 102,17 \\ 95,81 & 95,64 & 101,15 & 101,97 \\ 108,66 & 104,56 & 97,63 & 112,91\end{array}$

$\begin{array}{rrrr}100,74 & 94,43 & 96,51 & 99,59 \\ 101,61 & 97,56 & 94,01 & 96,98 \\ 91,29 & 111,27 & 105,57 & 95,39\end{array}$




\section{Raw data for sup figure $2 B$}


ADCC+ plate 1 berekening killing CTB

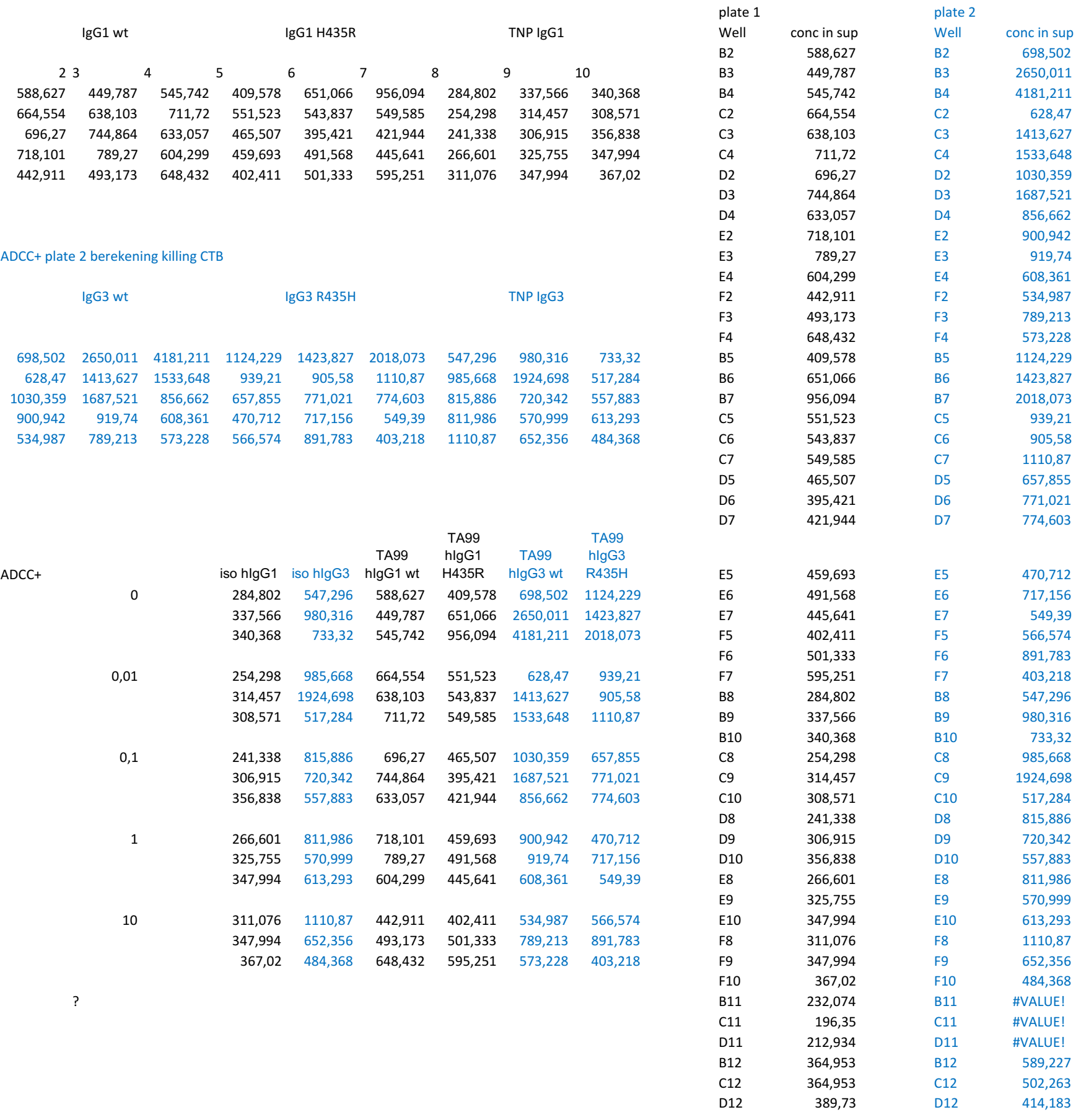

